\title{
Resistance
}

Intervirology

\section{Hepatitis B Virus Drug Resistance Tools: One Sequence, Two Predictions}

\author{
Maria Neumann-Fraune ${ }^{a}$ Bastian Beggel ${ }^{b}$ Rolf Kaiser ${ }^{a}$ Martin Obermeier ${ }^{c}$ \\ anstitute of Virology, University of Cologne, Cologne, ${ }^{b}$ Department of Computational Biology and Applied \\ Algorithms, Max Planck Institute for Informatics, Saarbrücken, and ${ }^{\mathrm{C}}$ Medical Center for Infectious Diseases Berlin \\ (MIB), Berlin, Germany
}

\section{Key Words}

Hepatitis B virus resistance - Escape mutations - Genotypic testing $\cdot$ Bioinformatics $\cdot$ Interpretation system

\begin{abstract}
Drug resistance testing, genotype analysis, and the determination of immune and vaccine escape variants support personalized antiviral treatment for hepatitis B patients. As phenotypic drug resistance testing for hepatitis $B$ virus (HBV) is especially labor-intensive, due to the lack of simple cell culture systems, genotypic methods play a very pronounced role. The genetic structure of HBV allows the simultaneous analysis of two different genes by examination of a single region in the genome of HBV. Nevertheless, the overlapping open reading frames of the hepatitis B surface antigen ( $\mathrm{HBsAg}$ ) and the reverse transcriptase (RT) have to be interpreted separately. In diagnostic procedures, standard Sanger type sequencing (mostly performed as a dye-dideoxynucleotide terminator system) is still the most commonly used method. This allows using established techniques for interpreting those types of genetic information. Besides reviewing the foundation of drug resistance interpretation for HBV, different interpretation systems, either commercially available (TRUGENE, Abbott, ViroScore) or free to use (geno2pheno[HBV], HIV-GRADE HBV tool), are compared in this article.

(c) 2014 S. Karger AG, Basel
\end{abstract}

\section{Overlapping Open Reading Frames: HBV's Peculiarity and Its Consequence for Clinical Applications}

The natural course of hepatitis B virus (HBV) infection often shows long phases of massive replication activity and high viral loads. Despite having a genome coded in DNA within the virus particles, a necessary RNA intermediate in the replication cycle of HBV is transcribed by a reverse transcriptase (RT). In the enzymatic relevant regions, this polymerase shows high structural similarity to the RT of HIV [1]. In HBV as in HIV, the missing proofreading function of the RT in addition to a high replication rate causes multiple mutation events. However, in contrast to HIV, the variability of HBV's sequence is limited by the overlapping of the two open reading frames (ORFs) of the RT and the hepatitis B surface antigen (HBsAg): both proteins share one region on the genome. Consequently, protein sequence analysis should always include both ORFs because a mutation in one ORF may alter the protein coded by the other ORF as well [2]. A worst-case scenario would be a viable virus that carries both a resistance mutation in the RT ORF and an escape mutation in the HBsAg ORF. Although the overlapping ORF structure limits the variability, the restrictions are not too high. For example, HBV genomes of some pa-

\section{KARGER}

E-Mail karger@karger.com

www.karger.com/int
(C) 2014 S. Karger AG, Basel

0300-5526/14/0574-0232\$39.50/0
Dr. Rolf Kaiser

Institute for Virology, University of Cologne

Fürst-Pückler-Strasse 56

DE-50935 Cologne (Germany)

E-Mail rolf.kaiser@uk-koeln.de 
tients treated with the nucleotide analogue adefovir (ADF) have resistance mutations in the RT which simultaneously lead to stop codons in the HBsAg ORF. Replication of these HBV genomes is still possible; the most plausible explanation is that the lack of functional HBsAg in these genomes is complemented by viable other $\mathrm{HBV}$ genomes in the form of cccDNA (covalently closed circular DNA) that occur in the same cell [3] and supply expression of functional $\mathrm{HBsAg}$ in trans. HBV genome fragments encoding truncated HBsAg have been found in hepatocellular carcinomas and cells containing these fragments may induce tumors in humanized immunodeficient mice. Thus, occurrence of such therapy-induced mutants may be associated with a higher risk of hepatocellular carcinoma development [4].

\section{HBV Genotypes}

There are 9 different genotypes (A-I) of the hepatitis $B$ virus, which differ in at least $8 \%$ of their DNA sequence. Further, different subgenotypes can be distinguished and have $>4 \%$ divergence in their nucleotide sequence, although some researchers also consider $>3.5 \%$ as sufficient if there is strong boot strap support (see Kramvis [this issue, pp. 141-150]). The different genotypes and subgenotypes have typical geographical and ethnical distributions $[5,6,26]$.

For the treatment of the disease, inhibitors of the RT as well as interferon (IFN) are used [7]. While no differences in sensitivity to RT inhibitors associated with wildtype sequence variety could be observed, there is an association of the genotype to the IFN treatment response. Patients with genotypes A and B have a higher sustained success rate with IFN than those with genotypes $C$ and D. On this basis, genotyping prior to treatment with IFN is recommended in the German guidelines for the therapy of HBV infections $[7,8]$.

\section{HBV Resistance}

In addition to IFN, 5 directly active drugs are approved for the treatment of hepatitis B infection and allow several ways of optimal treatment. ADF, entecavir (ETV), lamivudine (3TC), telbivudine (Tlb), and tenofovir (TDF) are either nucleoside or nucleotide analogues, of which 3TC and TDF are also used in HIV therapy. This treatment efficacy is based on the structural similarity of HBV and HIV RT. The selection of resis- tant virus variants is a major challenge in the treatment of hepatitis B. After 1 year of treatment with 3TC, 14$32 \%$ of patients carry resistant variants, increasing to $80 \%$ after 4 years [9]. After the approval of ADF, many patients with failing 3TC treatment underwent a therapy switch to ADF. Admittedly, 20\% did not respond from the beginning and $29 \%$ showed resistance to ADF after 5 years of treatment $[9,10]$. ETV had low resistance rates in therapy-naïve patients, namely $0.2 \%$ after 1 year and $1.2 \%$ after 5 years, in contrast to $57 \%$ of patients pretreated with $3 \mathrm{TC}$ after 5 years $[9,11]$. To date, TDF is the only drug for which no resistance has been reported since its approval in 2008, and this has been recently confirmed for 6 years of therapy [12]. It was reported that patients with ADF failure do not respond to TDF as completely as patients with another pretreatment, but this lower sensitivity does not reach the level of clinically relevant resistance [13].

\section{Escape Mutations}

To date, it is not clear which factors are decisive for a chronic or a resolving course of an acute HBV infection. In highly active persistent infections, the immune system is quasi-tolerant to the viral antigens except for the formation of nonprotective antibodies against the core antigen. In resolving cases, gene expression and replication of $\mathrm{HBV}$ is controlled by an active cellular immune response so that ultimately no free virus will be detected in the plasma. However, low levels of HBV cccDNA usually remain in the nuclei of hepatocytes and may replicate at very low levels. These occult HBV infections may reactivate expression of $\mathrm{HBV}$ gene products and restart enhanced replication if the immune competence of the patient is impaired. The reactivated virus may have signs of T-cell escape, but occasionally only a wild-type virus reappears.

Neutralizing antibodies against the HBsAg direct a high selective pressure on the $\mathrm{S}$ gene and can induce selection of escape variants. The selection of such escape variants can be detected in $40 \%$ of all patients receiving long-term passive immunization with hepatitis B immunoglobulin [14], but they are also often found in reactivated $\mathrm{HBV}$ infections [15]. Mutations of HBsAg causing immune escape can also be the result of changes in the RT due to antiviral therapy because of the overlapping reading frames [16]. Those escape mutations can impair protection by active vaccination or the natural immune response, and may weaken detection of HBsAg in diagnostic assays. The development of stop codon mutations in 
Table 1. Relevant RT mutations used for interpretation by the TRUGENE HBV genotyping assay software

\author{
A181T \\ A181IV + M204I \\ $\mathrm{A} 181 \mathrm{~V} / \mathrm{T}$ \\ $\mathrm{I} 169 \mathrm{~T}+\mathrm{V} 173 \mathrm{~L}+\mathrm{L} 180 \mathrm{M}+\mathrm{T} 184 \mathrm{G}+\mathrm{S} 202 \mathrm{I}+\mathrm{M} 204 \mathrm{~V}$ \\ $\mathrm{I} 169 \mathrm{~T}+\mathrm{V} 173 \mathrm{~L}+\mathrm{L} 180 \mathrm{M}+\mathrm{M} 204 \mathrm{~V}+\mathrm{M} 250 \mathrm{~V}$ \\ $\mathrm{I} 169 \mathrm{~T}+\mathrm{V} 173 \mathrm{~L}+\mathrm{L} 180 \mathrm{M}+\mathrm{M} 204 \mathrm{~V}$ \\ $\mathrm{L} 180 \mathrm{M}+\mathrm{M} 204 \mathrm{~V} / \mathrm{I} / \mathrm{S}$ \\ $\mathrm{L} 180 \mathrm{M}+\mathrm{A} 194 \mathrm{~T}+\mathrm{M} 204 \mathrm{~V}$ \\ $\mathrm{L} 180 \mathrm{M}+\mathrm{M} 204 \mathrm{~V} / \mathrm{I}$ \\ L180V/I + M204I (non-A genotype) \\ M204I \\ $\mathrm{N} 236 \mathrm{~T}$ \\ Q215S + L180M + M204V \\ $\mathrm{T} 184 \mathrm{~S}+\mathrm{L} 180 \mathrm{M}+\mathrm{M} 204 \mathrm{~V}$ \\ $\mathrm{V} 173 \mathrm{~L}+\mathrm{L} 180 \mathrm{M}+\mathrm{M} 204 \mathrm{~V}$ \\ V214A/Q215S \\ Various combinations of mutations at codons 184, 202, and 250
}

Data according to manufacturer's protocol.

the HBsAg frame can be a further consequence. Stop codons lead to the expression of truncated HBsAg that is no longer secreted anymore and stored in the ER. The resulting ER stress plus the cytosolic localization of the preS transcription activator induce the myc pathway, leading to a higher risk of hepatocellular carcinoma development [17].

\section{Prediction Systems: Interpretation Tools}

As for HIV resistance, several tools are available for resistance testing in HBV - either commercially or freely accessible via the internet. A state-of-the-art interpretation system should analyze sequences of both HBV proteins, the RT, and HBsAg of all genotypes.

\section{Commercial Systems}

The TRUGENE HBV Genotyping Assay (Siemens Healthcare) allows the identification of the HBV genotype and HBV mutations at the same time using direct sequence analysis (rules set see table 1). It describes itself as 'a fully integrated HBV genotyping solution: hardware, chemistry, software and report' as it provides a sequence analyzer, reagents for PCR, and sequencing reactions, as well as alignment and interpretation software.

The Abbott HBV Sequencing Assay provides reagents for amplification and sequencing for Life Technologies ${ }^{\mathrm{TM}}$
Table 2. HIV-GRADE HBV tool set of rules for interpreting hepatitis $\mathrm{B}$ resistance and $\mathrm{HBsAg}$ escape

\begin{tabular}{l} 
RT inhibitors \\
Lamivudine \\
Resistance \\
204ISV \\
Possible resistance \\
$\quad$ 180M, 173L, 80VI, 181TV \\
Telbivudine \\
Resistance \\
204IV \\
Possible resistance \\
$\quad$ 180M, 80VI, 181TV \\
Adefovir 1 \\
Resistance \\
236T, 233V, A181STV \\
Entecavir ${ }^{2}$ \\
Resistance \\
204IV and 1 of (169T, 184AGILS, 202CGI, 250IV) \\
Possible resistance \\
1 of (169T, 184AGILS, 202CGI, 250IV); 204IV and 1 of \\
$\quad$ (173L, 180M) \\
\hline Tenofovir \\
Resistance \\
Not known \\
Possible resistance \\
194T, 181V, 236T \\
HBs-antigen escape \\
Anti-HBs escape \\
1 of (100C, 101HY, 105L, 109RVHI, 110MRHIV, 114R, 117T, \\
118KRSP, 119R, 120AELPQSTR, 121SY, 123AN, 124RY, \\
126NSVIT, 127RSTL, 128V, 129HKPR, 130DRN, 131DISK, \\
cording to the manufacturer's instructions. \\
132APF, 133ILTV, 134HNRSVT, 137RSWY, 139S, 140S, \\
141EIRW, 142LRS, 143LPA, 144AEGH, 145AKLR, 146DS, 147ST) \\
\hline
\end{tabular}

(Applied Biosystems ${ }^{\mathrm{TM}}$ ) capillary sequence analyzers. It targets the RT region of the HBV viral genome and is also capable of detecting genotype and RT resistance mutations in the same assay. Interpretation of the derived sequences can be performed with the commercial SeqHepB [18] or with freely available interpretation systems like geno2pheno or HIV-GRADE (see below).

ViroScore $^{\circledR}$ (ABL SA Group) provides the SeqHepB interpretation system [18]. SeqHepB is a genotype interpretation, database, and analysis tool, and proposes more 
than 7 different drug resistance interpretation algorithms issued from expert groups. It is capable of determining HBV RT mutations and performs HBV drug resistance interpretation for ADF, ETV, 3TC, Tlb, and TDF, as well as HBsAg mutation determination and especially vaccine escape assessment. Although the functionalities currently available are limited to Sanger sequencing, ABL is currently adapting it to handle next-generation sequencing HBV data, and also to perform hepatitis delta virus genotyping and liver cancer susceptibility assessment.

\section{Noncommercial Systems}

Several prediction tools are capable of interpreting both protein sequences and can also be used with the output of the commercial assays as well as in-house assays.

Amplification and sequence analysis can, for example, be performed by using a protocol described by Schildgen et al. [19], and can be improved by additional oligonucleotides (HBrt-fw: gtcacttccggagactactgttgttagacgacg, HBrt-rev: gcgcatcggtccggcagatgagaaggc and MHL-fw: gtcactcatatgctcttcatcetgctgctatgcc, HBsAg-fw: gtcactggtaccatggagagcacaacatcaggattc, HBsAg-rev: gcgcataagcttttcaaatgtatacccaaagacaaaag) [20].

geno2pheno[HBV] offers state-of-the-art sequence analysis of the HBV genome. Special attention has been paid to create easy to use and well-structured input and output forms, in which the user can submit and analyze up to 8 sequences simultaneously. Upon submission, each input sequence is aligned against an HBV consensus sequence of the RT domain. Subsequently, the HBV genotype $(\mathrm{A}-\mathrm{H})$ and $\mathrm{HBV}$ subgenotype $(\mathrm{A} 1-\mathrm{F} 2)$ are inferred using 325 reference sequences [5]. geno2pheno[HBV] implements a genotyping methodology that can identify and genotype inter- and intragenotype dual infections based on population-based sequencing data when multiple viral strains are visible in the input sequence [21]. The aligned nucleotide sequence is translated to the amino acid level with respect to both the RT ORF and the HBsAg ORF. All polymorphisms with respect to both reading frames are extracted. Using the list of mutations in the RT ORF, an expert system based on a set of Boolean expressions computes the resistance prediction for the $\mathrm{HBV}$ drugs 3TC, ADF, ETV, TDF, and Tlb. Prediction results are staged in four categories, namely susceptible, limited susceptibility, partly resistant, and resistant. The rules are based on literature research and incorporate recent research results. Similarly, the amino acid sequence of HBsAg is compared against a comprehensive list of escape mutations, which was crafted from the literature which is listed in the tool (www.genafor.org/services.php). The

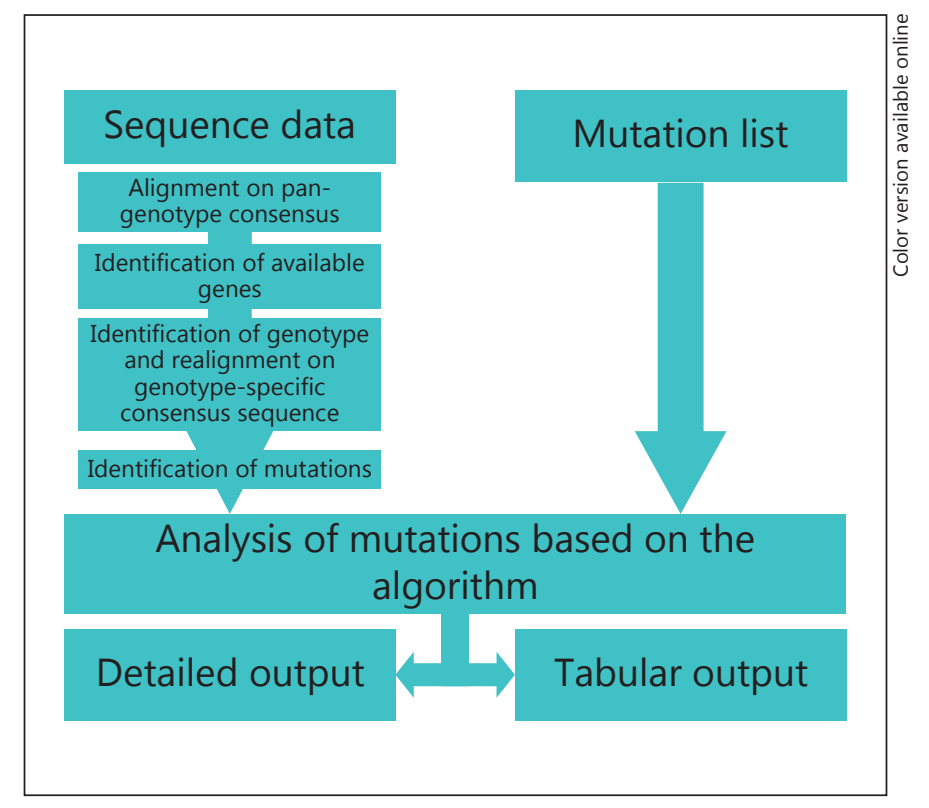

Fig. 1. Workflow of the HIV-GRADE HBV tool.

output includes the prediction of the mode of escape (vaccine, immune, and/or detection) for each relevant mutation, including a staging of the impact of each mutation (high impact, medium impact, secondary mutation). geno2pheno[HBV] offers the export of the analysis result as a PDF file, which might be used to archive the analysis or for communication purposes.

The HIV-GRADE HBV tool is an adaptation of the HIV-GRADE [22] tool to HBV. The HIV-GRADE tool itself is based on the source code from the Stanford $\mathrm{HIVdb}$ tools [23]. In brief, the nucleic acid sequence is aligned to a pan-genotype consensus sequence, and then realigned to a genotype-specific consensus sequence after comparing the homology to different representative sequences and genotype-specific consensus sequences. Differences to this closest genotype consensus sequence are extracted and used for further analysis. The applied sequence information was gathered by an extensive search of genotyped sequences in Genbank [24]. The differences to the consensus sequence are then classified by the rules in the HIV-GRADE HBV tool (fig. 1). The rule set itself is described in XML format based on the algorithm specification interface developed by the Stanford HIVdb group. Rules are based on extensive literature research and own clinical experience by the members of the HIV-GRADE society (see table 2) [25]. Necessity of rule changes is discussed twice a year at the regular meetings of the HIV-GRADE society. 


\section{Conclusion}

The prediction systems currently available are rulebased interpretations. In contrast to HIV, machinelearning techniques have not been implemented into available tools yet. Phenotypic data from the assay described in this issue (see Glebe and Geipel [this issue, pp. 134-140]) have not reached the amount necessary for machine-learning approaches to replace the rule-based systems. While there are plenty of clinical data for $\mathrm{Tlb}$,
3TC, and ADF, it is still a challenge to assign a clinical cutoff for ETV despite clear description of resistance mutations. For TDF, no resistance mutations have been verified in clinical samples so far. With the described free and commercial tools, systems are available that allow the interpretation of one HBV sequence in both ORFs. Personalized treatment strategies are simplified with these methods, allowing highly effective treatment for hepatitis $\mathrm{B}$ and reducing the risk of emerging resistance and escape of HBV.

\section{References}

-1 Das K, Xiong X, Yang H, et al: Molecular modeling and biochemical characterization reveal the mechanism of hepatitis $B$ virus polymerase resistance to lamivudine (3TC) and emtricitabine (FTC). J Virol 2001;75: 4771-4779.

2 Warner N, Locarnini S, Kuiper M, et al: The L80I substitution in the reverse transcriptase domain of the hepatitis B virus polymerase is associated with lamivudine resistance and enhanced viral replication in vitro. Antimicrob Agents Chemother 2007;51:2285-2292.

$>3$ Zhang YY, Zhang BH, Theele D, Litwin S, Toll E, Summers J: Single-cell analysis of covalently closed circular DNA copy numbers in a hepadnavirus-infected liver. Proc Natl Acad Sci USA 2003; 100:12372-12377.

4 Mitri D, Stella M, Cassini R, Bernardi M: Hepatitis B virus-related hepatocarcinogenesis: molecular oncogenic potential of clear or occult infections. Eur J Cancer 2010;46:21782186.

5 Norder H, Couroucé AM, Coursaget P, et al: Genetic diversity of hepatitis B virus strains derived worldwide: genotypes, subgenotypes, and HBsAg subtypes. Intervirology 2004;47: 289-309.

6 Yu H, Yuan Q, Ge SX, et al: Molecular and phylogenetic analyses suggest an additional hepatitis B virus genotype 'I'. PLoS ONE 2010; 5:e9297.

$\checkmark 7$ Cornberg M, Protzer U, Petersen J, et al: Aktualisierung der S 3-Leitlinie zur Prophylaxe, Diagnostik und Therapie der Hepatitis-B-Virusinfektion. Z Gastroenterol 2011;49:871930.
8 Cooksley WG: Do we need to determine viral genotype in treating chronic hepatitis $\mathrm{B}$ ? J Viral Hepat 2010;17:601-610.

$\checkmark 9$ Zoulim F, Locarnini S: Hepatitis B virus resistance to nucleos(t)ide analogues. Gastroenterology 2009;137:1593-1608.e2.

10 Hadziyannis SJ, Tassopoulos NC, Heathcote EJ, et al: Long-term therapy with adefovir dipivoxil for HBeAg-negative chronic hepatitis B for up to 5 years. Gastroenterology 2006; 131:1743-1751.

$>11$ Tenney DJ, Rose RE, Baldick CJ, et al: Longterm monitoring shows hepatitis $\mathrm{B}$ virus resistance to entecavir in nucleoside-naive patients is rare through 5 years of therapy. $\mathrm{He}$ patology 2009;49:1503-1514.

12 Kitrinos KM, Corsa A, Liu Y, et al: No detectable resistance to tenofovir disoproxil fumarate after 6 years of therapy in patients with chronic hepatitis B. Hepatology 2014;59:434442.

$>13$ Van Bömmel F, de Man RA, Wedemeyer H, et al: Long-term efficacy of tenofovir monotherapy for hepatitis B virus-monoinfected patients after failure of nucleoside/nucleotide analogues. Hepatology 2010;51:73-80.

14 Weber B: Genetic variability of the S gene of hepatitis B virus: clinical and diagnostic impact. J Clin Virol 2005;32:102-112.

15 Gerlich WH, Bremer C, Saniewski M, et al: Occult hepatitis B virus infection: detection and significance. Dig Dis 2010;28:116-125.

16 Sloan RD, Ijaz S, Moore PL, Harrison TJ, Teo CG, Tedder RS: Antiviral resistance mutations potentiate hepatitis B virus immune evasion through disruption of its surface antigen a determinant. Antivir Ther 2008;13: 439-447.

17 Lai MW, Yeh CT: The oncogenic potential of hepatitis B virus rtA181T/surface truncation mutant. Antivir Ther 2008;13:875-879.
18 Yuen LK, Ayres A, Littlejohn M, et al SeqHepB: a sequence analysis program and relational database system for chronic hepatitis B. Antiviral Res 2007;75:64-74.

19 Schildgen O, Sirma H, Funk A, et al: Variant of hepatitis B virus with primary resistance to adefovir. N Engl J Med 2006;354:1807-1812.

20 Neumann-Fraune M, Beggel B, Pfister H, Kaiser R, Verheyen J: High frequency of complex mutational patterns in lamivudine resistant hepatitis B virus isolates. J Med Virol 2013;85: 775-779.

21 Beggel B, Neumann-Fraune M, Döring M, et al: Genotyping hepatitis B virus dual infections using population-based sequence data. J Gen Virol 2012;93:1899-1907.

22 Obermeier M, Pironti A, Berg T, et al: HIVGRADE: a publicly available, rules-based drug resistance interpretation algorithm integrating bioinformatic knowledge. Intervirology 2012;55:102-107.

23 Liu TF, Shafer RW: Web resources for HIV type 1 genotypic-resistance test interpretation. Clin Infect Dis 2006;42:1608-1618.

24 Benson DA, Karsch-Mizrachi I, Lipman DJ, Ostell J, Wheeler DL: GenBank. Nucleic Acids Res 2005;33:D34-D38.

25 HIV-GRADE. http://www.hiv-grade.de/cms/ grade/homepage.html (accessed June 11, 2012).

26 Tatematsu K, Tanaka Y, Kurbanov F, Sugauchi F, Mano S, Maeshiro T, Nakayoshi T, Wakuta M, Miyakawa Y, Mizokami M: A genetic variant of hepatitis B virus divergent from known human and ape genotypes isolated from a Japanese patient and provisionally assigned to new genotype J. J Virol 2009; 83:10538-10547. 